\title{
On the equivalence of Clauser-Horne and Eberhard inequality based tests
}

\author{
Andrei Khrennikov*1, Sven Ramelow ${ }^{2,3,4}$, Rupert Ursin ${ }^{2}$, \\ Bernhard Wittmann ${ }^{2,3}$, Johannes Kofler ${ }^{5}$, and Irina Basieva ${ }^{6}$ \\ ${ }^{1}$ International Center for Mathematical Modeling, in Physics, \\ Engineering, Economics, and Cognitive Science, Linnaeus \\ University, Växjö-Kalmar, Sweden \\ ${ }^{2}$ Institute for Quantum Optics and Quantum Information \\ Vienna (IQOQI), Austrian Academy of Sciences, \\ Boltzmanngasse 3, Vienna, Austria \\ ${ }^{3}$ Quantum Optics, Quantum Nanophysics, Quantum \\ Information, University of Vienna, Faculty of Physics, \\ Boltzmanngasse 5, Vienna, Austria \\ ${ }^{4}$ Cornell University, 271 Clark Hall, 142 Science Dr., Ithaca, \\ 14853 NY, USA \\ ${ }^{5}$ Max Planck Institute of Quantum Optics (MPQ), \\ Hans-Kopfermann-Str. 1, 85748 Garching, Germany \\ ${ }^{6}$ Prokhorov General Physics Institute, Vavilov str. 38D, \\ Moscow, Russia
}

June 3, 2022

\begin{abstract}
Recently, the results of the first experimental test for entangled photons closing the detection loophole (also referred to as the fair sampling loophole) were published (Vienna, 2013). From the theoretical viewpoint the main distinguishing feature of this long-aspired
\end{abstract}

*email: Andrei.Khrennikov@lnu.se 
experiment was that the Eberhard inequality was used. Almost simultaneously another experiment closing this loophole was performed (Urbana-Champaign, 2013) and it was based on the Clauser-Horne inequality (for probabilities). The aim of this note is to analyze the mathematical and experimental equivalence of tests based on the Eberhard inequality and various forms on the Clauser-Horne inequality. The structure of the mathematical equivalence is nontrivial. In particular, it is necessary to distinguish between algebraic and statistical equivalence. Although the tests based on these inequalities are algebraically equivalent, they need not be equivalent statistically, i.e., theoretically the level of statistical significance can drop under transition from one test to another (at least for finite samples). Nevertheless, the data collected in the Vienna-test implies not only a statistically significant violation of the Eberhard inequality, but also of the Clauser-Horne inequality (in the ratio-rate form): for both a violation $>60 \sigma$.

\section{Introduction}

Experimental realization of a loophole-free test for Bell [1] inequalities will have impact both for quantum foundations and quantum technologies. In both cases the present situation, e.g., in quantum cryptography [2] and quantum random generators [3] (see also [4] for discussion) is unsatisfying from the scientific viewpoint. To experimentally falsify local realism, a so called loophole-free Bell experiment will have to be accomplished successfully 1 This was not claimed so far in any of the reported experiments. Up to now, space-like separation of measurements and basis choices has been accomplished in the pioneering experiments of Aspect et al. [5, 6] and Weihs et al. 7], closing the so-called locality loophole.2 These experimental tests were based on the Clauser-Horne-Shimony-Holt (CHSH) inequality [9]. For this inequality to falsify local realism one must either approach very high total detection efficiency (which includes the optical losses of the setup and the efficiency of the detectors) $\eta=82.8 \%$, or proceed under an assumption

\footnotetext{
${ }^{1}$ We remark that a priori one still cannot exclude the possibility that in the final loophole free experiment the Bell inequality would be satisfied. In such (very improbable) case, since quantum theory predicts that, for the state under preparation, Bell's inequality has to be violated, the experiment would imply rejection of the quantum model. Thus the Bell test can also be considered as an attempt to falsify quantum mechanics. (At the initial stage of Bell experimentation expectation that quantum mechanics would be falsified was quite common.)

${ }^{2}$ Although we discuss only Bell tests for entangled photons, it is relevant that the first closure of the detection loophole was achieved with massive particles [8].
} 
to circumvent the loss - the so-called fair sampling assumption, see section 2 for discussion. It has been shown that using unfair sampling at sufficiently low detection efficiencies, very simple local models with hidden variables can violate the CHSH-inequality, e.g., [2], [10]- [18]. Thus in the CHSHframework, approaching very high detection efficiency is the only possible way to experimentally falsify the classical world view of local realism. In spite of technological progress and the existence of detectors whose efficiency nears unity, (e.g., TES detectors with efficiency around 95\% [19]), it is very challenging to approach the required total detection efficiency.

In 1974, Clauser and Horne [10] proposed a new inequality that is not based on the fair sampling assumption. This inequality is expressed in terms of probabilities and we shall call it the $\mathrm{CH}$-inequality for probabilities:

$$
p\left(\alpha_{1}, \beta_{1}\right)+p\left(\alpha_{1}, \beta_{2}\right)+p\left(\alpha_{2}, \beta_{1}\right)-p\left(\alpha_{2}, \beta_{2}\right) \leq p^{A}\left(\alpha_{1}\right)+p^{B}\left(\beta_{1}\right),
$$

where $p(\alpha, \beta)$ and $p^{A}(\alpha), p^{B}(\beta)$ are probabilities for coincidence and single counts 3 , respectively; see section 4 for more detail.

In this paper we shall discuss various forms of the $\mathrm{CH}$-inequality, see section 4 (see also the review of Clauser and Shimony [11] and the Stanford encyclopedia paper of Shimony [20] for details). Therefore we address each form with the corresponding label. However, we restrict our considerations to the class of the $\mathrm{CH}$-inequalities not based on experimentally untestable auxiliary assumptions. Thus we shall not consider the $\mathrm{CH}$-inequality whose derivation is based on the "no-enhancement assumption" [10]: if an analyzer is removed from one of the paths, the resulting probability of detection is at least as great as with an analyzer.

To determine probabilities in (1), one has to know the total number of emitted pairs of photons. As was pointed out by Clauser and Horne [10], see also the review of Clauser and Shimony [11] for extended discussion, it is practically impossible to determine this number experimentally. To escape this problem, it was proposed [11] to exclude the total number of emitted pairs from consideration by considering a version of the $\mathrm{CH}$-inequality in the form of ratio of detection counts rates:

$$
T=\frac{R\left(\alpha_{1}, \beta_{1}\right)+R\left(\alpha_{1}, \beta_{2}\right)+R\left(\alpha_{2}, \beta_{1}\right)-R\left(\alpha_{2}, \beta_{2}\right)}{R^{A}\left(\alpha_{1}\right)+R^{B}\left(\beta_{1}\right)} \leq 1,
$$

where $R(\alpha, \beta)$ and $R^{A}(\alpha), R^{B}(\beta)$ are coincidence and single rates, respectively; see section 4 for details.

\footnotetext{
3 "Single counts" are defined as all counts registered on one side for a given setting.

${ }^{4}$ We remark that an important implicit assumption of applicability of this inequality is the assumption of (statistically) constant production rate for pairs of photons, see also section 4 for discussion.
} 
For the CH-inequality for probabilities (1), Clauser and Horne [10] formulated the restrictions on the experimental setup in a straightforward way: first finding the "optimal angles" $(\alpha, \beta)$ and then calculating other experimental parameters, namely the degree of entanglement and detection efficiency, to violate the inequality, see equations (5) and (6) in [10]. It can be shown that this procedure leads to very high detection efficiency. To violate the $\mathrm{CH}-$ inequality for probabilities, the detection efficiency has to be at least $82.8 \%$, see [2, 21].

In 22] Eberhard proposed a different approach by jointly optimizing all aforementioned parameters. He derived a new Bell inequality which we will abbreviate by "E-inequality":

$$
\begin{gathered}
J \equiv n_{\text {oe }}\left(\alpha_{1}, \beta_{2}\right)+n_{\text {ou }}\left(\alpha_{1}, \beta_{2}\right)+n_{\text {eo }}\left(\alpha_{2}, \beta_{1}\right)+n_{\text {uo }}\left(\alpha_{2}, \beta_{1}\right)+n_{\text {oo }}\left(\alpha_{2}, \beta_{2}\right) \\
-n_{\text {oo }}\left(\alpha_{1}, \beta_{1}\right) \geq 0,
\end{gathered}
$$

where $n_{x y}\left(\alpha_{i}, \beta_{j}\right)$ is the number of pairs detected in a given time period for settings $\alpha_{i}, \beta_{j}$ with outcomes $x, y=o, e, u$ and the outcomes $(o)$ and $(e)$ correspond to detections in the ordinary and extraordinary beams, respectively, and the event that photon is undetected is denoted by the symbol $(u)$. We point to the main distinguishing features of the E-inequality:

a) derivation without the fair sampling assumption (and without the noenhancement assumption);

b) taking into account undetected photons;

c) background events are taken into account;

d) the linear form of presentation (non-negativity of a linear combination of coincidence and single rates).

The latter feature (which is typically not emphasized in the literature) is crucial to find a simple procedure of optimization of experimental parameters and, hence, it makes the E-inequality the most promising experimental test to close the detection loophole and to reject local realism without the fair sampling assumption. Eberhard's optimization has two main outputs which play an important role in the experimental design:

E1). It is possible to perform an experiment without fair sampling assumption for detection efficiency less than $82,8 \%$. Nevertheless, detection efficiency must still be very high, at least $66.6 \%$ (in the absence of background).

E2). The optimal parameters correspond to non-maximally entangled states.

In 2013, the possibility to proceed with overall efficiencies lower than $82.8 \%$ (but larger than $66.6 \%$ ) was explored for the E-inequality and the 
first experimental test ("the Vienna test") closing the detection loophole was published [23], for more detailed presentation of statistical data see also [24], 25].

Almost simultaneously another experiment closing the detection loophole was performed [26], [27] based on the probability version of the $\mathrm{CH}$-inequality [10], see (11).

In this note we analyze the mathematical and experimental equivalence of the tests based on the E-inequality (3), and the $\mathrm{CH}$-inequality in the ratiorate form (2) - in fact, its modification for the ratio of detection counts, see section 4, the inequality (9). In particular, one has to distinguish between algebraic and statistical equivalence (and for the latter, the cases of finite and infinite samples). Although these inequalities are (trivially) algebraically equivalent, (see section 3.2), the tests based on them need not be equivalent statistically (for finite samples), i.e., theoretically the level of statistical significance can change essentially under transition from one test to another, section 3.4. Nevertheless, the data collected in the Vienna test [23] implies not only the statistically significant violation of the E-inequality, but also of the $\mathrm{CH}$-inequality for ratio of detection counts and, hence, for ratio of detection rates: for both a violation $>60 \sigma$.

One of the aims of this note is to determine confidence intervals from the statistics of the data collected in [23]. We remark that if one does not assume that data is Gaussian, then it is impossible to determine the confidence interval exactly with the aid of the standard deviation. However, it is possible to estimate it by using the Chebyshev inequality, see, e.g., [28]. This inequality is applicable under the most conservative (worst case) assumption - namely that the dispersion is finite. Although the Chebyshev inequality gives only rough estimates of probabilities, in our case (for the data collected in the Vienna test) it is sufficiently powerful to estimate confidence intervals showing that the hypothesis about the local realistic description of the Vienna data must be rejected.

\section{Fair sampling assumption}

The fair sampling assumption plays a crucial role in justification of tests based on the CHSH-inequality. In [9] it was stated as

"if a pair of photons emerges from [the polarizers], the probability of their joint detection is independent of [polarizer orientations]."

Extended discussions on the role of this assumption in the resolution of the classical-quantum dilemma can be found in the papers of Pearle [2] and 
Clauser and Horne [10] and Aspect's PhD thesis [6], see also Aspect's "naive experimentalist presentation" of Bell's tests [29]. Later the fair sampling assumption was analyzed in detail in the $\mathrm{PhD}$ theses of Larsson [30] and Adenier [31.

The fair sampling assumption is not made in a Bell test based on the E-inequality or any $\mathrm{CH}$-inequality. We also remark that the no-enhancement assumption is not present in the list of assumptions for the derivation of the E-inequality, see [22], the assumptions (i)-(iii).

Finally, we remark that in this paper we do not discuss two other important loopholes, the coincidence-time loophole (and the role of space-time in the Bell argument in general, cf. [32]- 40], [17]) and the freedom-of-choice loophole [41] (and its relation to the impossibility to use the conventional model of classical probability theory, the Kolmogorov model, 1933, see [42][47, [17], [35, 36]).

\section{On equivalence of the E-inequality to the $\mathrm{CH}$-inequalities}

\subsection{E-inequality}

We follow Eberhard [22]: Photons are emitted in pairs $(a, b)$. Under each measurement setting $(\alpha, \beta)$, the events in which the photon $a$ is detected in the ordinary and extraordinary beams are denoted by the symbols $(o)$ and $(e)$, respectively, and the event that it is undetected is denoted by the symbol $(u)$. The same symbols are used to denote the corresponding events for the photon $b$. Therefore for the pairs of photons there are nine types of events: $(o, o),(o, u),(o, e),(u, o),(u, u),(u, e),(e, o),(e, u)$, and $(e, e)$. (We remark that originally Eberhard considered the experiment with four detectors. In [23] his experimental design was modified to proceed with only two detectors. We shall come back to this point in section 3.2.)

Under the conditions of locality, realism and statistical reproducibility the inequality (3), see section 1, was derived.

\section{$3.2 \quad$ Algebraic equivalence}

As was mentioned in section 3.1, originally Eberhard derived his inequality for the four-detectors experiment, one detector at each output of two PBSs. In [23] it was shown that the "e-outputs" can be eliminated from the Einequality (3), i.e., the four-detectors experimental design can be transformed into the two-detectors design corresponding to detection of only "o-outputs". 
In this section we demonstrate that the latter version of the E-inequality is algebraically equivalent to the $\mathrm{CH}$-inequalities in various forms: for probabilities, ratio of probabilities, ratio of rates, and ratio of detection counts.

As was pointed out [23], the E-inequality can be transformed into the following inequality:

$-n_{\text {oo }}\left(\alpha_{1}, \beta_{1}\right)+S_{o}^{A}\left(\alpha_{1}\right)-n_{\text {oo }}\left(\alpha_{1}, \beta_{2}\right)+S_{o}^{B}\left(\beta_{1}\right)-n_{\text {oo }}\left(\alpha_{2}, \beta_{1}\right)+n_{\text {oo }}\left(\alpha_{2}, \beta_{2}\right) \geq 0$,

where $S_{o}^{A}\left(\alpha_{1}\right)$ and $S_{o}^{B}\left(\beta_{1}\right)$ are numbers of single counts in the $o$-channels for "Alice" and "Bob", in settings $\alpha_{1}$ and $\beta_{1}$ respectively. To match with the $\mathrm{CH}$-inequalities completely, we change the sign and collect singles terms in the right-hand side:

$$
n_{o o}\left(\alpha_{1}, \beta_{1}\right)+n_{o o}\left(\alpha_{1}, \beta_{2}\right)+n_{o o}\left(\alpha_{2}, \beta_{1}\right)-n_{o o}\left(\alpha_{2}, \beta_{2}\right) \leq S_{o}^{A}\left(\alpha_{1}\right)+S_{o}^{B}\left(\beta_{1}\right) .
$$

Then by dividing by the number of emitted pairs $N$ on both sides (and omitting the index "o") we obtain the $\mathrm{CH}$-inequality for probabilities 5 (Here we proceed under the assumption of statistically constant production rate for pairs of photons, cf. section 4.):

$$
p\left(\alpha_{1}, \beta_{1}\right)+p\left(\alpha_{1}, \beta_{2}\right)+p\left(\alpha_{2}, \beta_{1}\right)-p\left(\alpha_{2}, \beta_{2}\right) \leq p^{A}\left(\alpha_{1}\right)+p^{B}\left(\beta_{1}\right),
$$

where $p\left(\alpha_{i}, \beta_{j}\right)=n_{o o}\left(\alpha_{i}, \beta_{j}\right) / N, p^{A}\left(\alpha_{1}\right)=S_{o}^{A}\left(\alpha_{1}\right) / N, p^{B}\left(\beta_{1}\right)=S_{o}^{B}\left(\beta_{1}\right) / N$.

However, as pointed out by Clauser and Horne [10], this inequality suffers from the problem that the number $N$ and, hence, probabilities, are not welldetermined in an experiment. To solve this problem, (6) can be transformed into the inequality:

$$
T=\frac{p\left(\alpha_{1}, \beta_{1}\right)+p\left(\alpha_{1}, \beta_{2}\right)+p\left(\alpha_{2}, \beta_{1}\right)-p\left(\alpha_{2}, \beta_{2}\right)}{p^{A}\left(\alpha_{1}\right)+p^{B}\left(\beta_{1}\right)} \leq 1
$$

(We call this inequality [11] $C H$-inequality for ratio of probabilities, see Remark 1 later.) And finally $T$ can be represented as ratio of detection count rates:

$$
T=\frac{R\left(\alpha_{1}, \beta_{1}\right)+R\left(\alpha_{1}, \beta_{2}\right)+R\left(\alpha_{2}, \beta_{1}\right)-R\left(\alpha_{2}, \beta_{2}\right)}{R^{A}\left(\alpha_{1}\right)+R^{B}\left(\beta_{1}\right)} \leq 1,
$$

where $R(\alpha, \beta)$ and $R^{A}\left(\alpha_{1}\right), R^{B}\left(\beta_{1}\right)$ are coincidence and single rates, respectively. (Following Clauser and Shimony [11] we call this inequality $\mathrm{CH}$ inequality for ratio of detection count rates or simply the ratio-rates $\mathrm{CH}$ inequality, see again Remark 1.) This inequality is evidently equivalent to

\footnotetext{
${ }^{5}$ This inequality is sometimes referred simply as "CH-inequality", see Remark 1 for a short discussion on the terminology related to the papers of Clauser and Horne [10] and Clauser and Shimony [1].
} 
the following inequality in Eberhard's notation, i.e., with the total numbers of coincidences and single counts, instead of the rates:

$$
T=\frac{n\left(\alpha_{1}, \beta_{1}\right)+n\left(\alpha_{1}, \beta_{2}\right)+n\left(\alpha_{2}, \beta_{1}\right)-n\left(\alpha_{2}, \beta_{2}\right)}{S^{A}\left(\alpha_{1}\right)+S^{B}\left(\beta_{1}\right)} \leq 1 .
$$

(We call this inequality $\mathrm{CH}$-inequality for ratio of detection counts or simply the ratio-counts $\mathrm{CH}$-inequality, see again Remark 1.)

This inequality can be directly derived from (5) without relying on making any statements or assumptions about $N$. We can also proceed another way around and derive the E-inequality in the form (5) from the $\mathrm{CH}$-inequality in the form (9). Thus these two inequalities are algebraically equivalent. The problem of their statistical equivalence will be studied in section 3.4. And it is more complicated.

Remark 1. (On terminology) The CH-inequality for probabilities, see (6), is sometimes called simply the CH-inequality. At the same time, in the paper of Clauser and Shimony [11] this inequality was considered as just an intermediate step towards the ratio-rates $\mathrm{CH}$-inequality, see (8). Thus it would be natural to refer to the latter as the $\mathrm{CH}$-inequality. This terminology is used, e.g., by Shimony [20], who called the ratio-rates inequality, the $\mathrm{BCH}$ inequality, see also the experimental proposal of Fry and Walther [49]. The main source of referring to (6) as the $\mathrm{CH}$-inequality is that the material in the original CH-paper [10] was presented in a very compact form; in particular, the inequalities for ratios of probabilities and rates, see (17) and (8), were not written anywhere. The authors just remarked that the upper limit in (즈) can be experimentally testable without $N$ being known. This statement can be interpreted as simply the (algebraic) equivalence of the inequality (6) to the E-inequality (5) achieved with multiplication of the right- and left-hand sides of (6) by $N$ (again under the assumption of statistically constant production rate).

\subsection{Application of Chebyshev's inequality to data from the Vienna-test}

Consider statistical data that is normally distributed. The information about the mean value $\mu$ and the standard deviation $\sigma$ is sufficient to find the spread of these data relative to the number of standard deviations from the mean value. Denote the mean value and the standard deviation by the symbols $\mu$ and $\sigma$, respectively. It is known that $68 \%$ of these data are within $1 \sigma$ deviation from $\mu, 95 \%$ of the data are within $2 \sigma$-deviations from $\mu$, and approximately $99 \%$ of the data are within $3 \sigma$-deviation from $\mu$. 
However, if the statistical data set is not normally distributed, i.e., its density deviates from the bell shape, then a different amount could be within $k \sigma$-deviation, $k=1,2,3 \ldots$. In this case one can apply Chebyshev's inequality [48] - a powerful tool to get to know what fraction of the statistical data falls within a few standard deviations from the mean value. We recall that the Chebyshev theorem states that, for any random variable $\xi$ with finite second moment6, i.e., $E\left(|\xi|^{2}\right)<\infty$, where $E$ denotes the expectation value, and any positive number $c$,

$$
P(|\xi| \geq c) \leq \frac{E\left(|\xi|^{2}\right)}{c^{2}}
$$

Typically in applications one starts with a random variable $\eta$ and in (10) selects $\xi=\eta-\mu$, where $\mu=E(\eta)$ is the mean value of $\eta$. Thus

$$
P(|\eta-\mu| \geq c) \leq \frac{E\left(|\eta-\mu|^{2}\right)}{c^{2}}=\left(\frac{\sigma}{c}\right)^{2},
$$

where $\sigma$ is standard deviation of $\eta$. We remark that a violation with $c=k \sigma$ results in confidence probability for a violation of $\left(1-1 / k^{2}\right)$.

By using the values for the mean value and standard deviation calculated in [23] and by applying Chebyshev's inequality, we can find (without knowing the probability distribution exactly, but only assuming that the dispersion is finite, see footnote 9 ) that the E-inequality is violated statistically significantly.

Remark 2. It is important to present the procedure of calculation of the empirical values $m_{J}$ and $s_{J}$, which was used by Giustina et al. [23]: "After recording for a total of $300 \mathrm{~s}$ per setting we divided our data into 10-s blocks and calculated the standard deviation of the resulting 30 different $J$ values."

We consider random variables corresponding to the left-hand and righthand sides of the inequality (5):

$$
J_{\text {pair }}=n_{o o}\left(\alpha_{1}, \beta_{1}\right)+n_{o o}\left(\alpha_{1}, \beta_{2}\right)+n_{o o}\left(\alpha_{2}, \beta_{1}\right)-n_{o o}\left(\alpha_{2}, \beta_{2}\right)
$$

and

$$
J_{\text {single }}=S_{o}^{A}\left(\alpha_{1}\right)+S_{o}^{B}\left(\beta_{1}\right)
$$

Thus

$$
J=J_{\text {single }}-J_{\text {pair }} .
$$

Denote the mean value and standard deviation of the random variable $J$ by the symbols $\mu_{J}$ and $\sigma_{J}$, respectively 7 Thus $\mu_{J}=E(J)$ and $\sigma_{J}^{2}=E\left(J-\mu_{J}\right)^{2}$.

\footnotetext{
${ }^{6}$ In particular, the Chebyshev inequality is applicable to any bounded random variable.

${ }^{7}$ We remark that, since, for experimental runs of fixed duration, the number of emitted photon pairs is a bounded random variable, the random variable $J$ is bounded and, hence, $E|J|^{2}<\infty$. Therefore dispersion is well defined and the Chebyshev inequality is applicable.
} 
Our aim is to estimate the confidence interval for the mean. As always we shall use the statistical estimates of the mean and dispersion:

$$
\bar{J}=\frac{1}{L} \sum_{i=1}^{L} J_{i}, s_{J}^{2}=\frac{1}{L-1} \sum_{i=1}^{L}\left(J_{i}-\bar{J}\right)^{2}
$$

(in the experiment [23] $L=30$, this is the number of the 10-s blocks, see Remark 2, each block is used to calculate $J_{i}$, the number of pairs in each block is very large and in such a framework its exact number is not so important).

We shall also use the standard error of the mean (the standard deviation of the sample-mean's estimate of a population mean):

$$
\mathrm{SE}_{\overline{\mathrm{J}}}=\frac{\mathrm{S}_{\mathrm{J}}}{\sqrt{\mathrm{L}}}
$$

and the standard deviation of the mean:

$$
\mathrm{SD}_{\overline{\mathrm{J}}}=\frac{\sigma_{\mathrm{J}}}{\sqrt{\mathrm{L}}}
$$

We remark that $\mathrm{SE}_{\overline{\mathrm{J}}}$ decreases as $\sqrt{L}$ with increase of the size of the sample L. 8

By using the Chebyshev inequality we obtain:

$$
P\left(\left|\bar{J}-\mu_{J}\right| \geq c\right) \leq \frac{\mathrm{SD}_{\bar{J}}^{2}}{c^{2}}
$$

We proceed by using the standard error of the mean, instead of the standard deviation of the sample mean (in the formal mathematical presentation one has to use the correction related to the finite $L$, see [50], [51] for details):

$$
P\left(\left|\bar{J}-\mu_{J}\right| \geq c\right) \leq \frac{\mathrm{SE}_{\bar{J}}^{2}}{c^{2}}
$$

From [23] we take the values $\bar{J} \approx-4224$ and $\mathrm{SE}_{\bar{J}} \approx 61.23$. This is a $>60 \sigma$ violation, where $\sigma \equiv \mathrm{SE}_{\bar{J}}$. However, as was pointed out, for the statistical data from the Vienna test one cannot assume that these data are normally distributed. Therefore further analysis is needed. By using the the Chebyshev

\footnotetext{
${ }^{8}$ This makes intuitively also a lot of sense: the larger the sample one has the smaller the confidence interval for the mean value. At the same time one must not overestimate the role of getting a very small standard error of the mean. It mainly means that one was able to perform measurements for very long runs of the experiment and, in particular, to guarantee the stability of functioning of the source and the measurement devices.
} 
inequality we estimate the confidence interval corresponding to the confidence level $99.95 \%$. (In applied statistics already the level $95 \%$ is considered as sufficiently high.) Here $c \approx 2738$. Thus:

$$
P\left(m_{J} \in[\bar{J}-2738, \bar{J}+2738]\right)=P\left(m_{J} \in[-6962,-1486]\right) \geq 0.9995 .
$$

Thus the confidence that can be placed in the result of the Vienna-test is very high. The demonstrated violation of the E-inequality cannot be a matter of chance.

\subsection{Statistical (non-)equivalence}

Generally (i.e., without additional assumptions on probability distributions) the algebraic equivalence of two tests does not imply their statistical equivalence, at least for finite samples. In particular, the tests based on the Einequality and the CH-inequality in the form (9) are not statistically equivalent. The latter means that violation of one of them with $k \sigma$, where $k$ is sufficiently large, need not imply that another will be violated with the same $k$. It may happen that the significance of the violation changes essentially. This is a general statistical feature, i.e., it is not coupled rigidly with the two statistical tests under consideration, see appendix.

We remark that if the conditions of the central limit theorem are satisfied (in particular, for identically distributed independent random variables), then by using the $\delta$-method [52] (error propagation method) we one can prove the statistical equivalence of the E-test and the CH-test for $L \rightarrow \infty$. However, for finite $L$, in general it is true that by looking at different functions of statistics of interest and using the delta method, one can get any answer one likes. All of these answers are just approximations, and some approximations are better than others. As was pointed out, for $L \rightarrow \infty$, they give the same answer, but for fixed $L$ they all give different answers.

\subsection{Statistically significant violation of the CH-inequality (in the ratio form) for the Vienna-test}

Thus on the basis of purely theoretical arguments one cannot derive a statistically significant violation of the $\mathrm{CH}$-inequality in the form (9) from statistically significant violation of the E-inequality. One has to use again the experimental data.

In this section we apply Chebyshev's inequality to show that the statistical data collected in the Vienna-test also implies statistically significant violation of the (ratio-counts) $\mathrm{CH}$-inequality. Set $\mu_{T}=E(T)$ (the 
mean value of the random variable $T$ ) and $\sigma_{T}^{2}=E\left(T-\mu_{T}\right)^{2}$ (its dispersion). We shall use the statistical estimates of the mean and dispersion: $\bar{T}=\frac{1}{L} \sum_{i=1}^{L} T_{i}, s_{T}^{2}=\frac{1}{L-1} \sum_{i=1}^{L}\left(T_{i}-\bar{T}\right)^{2}$ (in the experiment [23] $L=30$, this is the number of the 10-s blocks, each block is used to calculate $T_{i}$ ). We also consider the standard deviation of the mean $\mathrm{SD}_{\overline{\mathrm{T}}}=\frac{\sigma_{\mathrm{T}}}{\sqrt{\mathrm{L}}}$ and and the standard error of the mean $\mathrm{SE}_{\overline{\mathrm{T}}}=\frac{\mathrm{s}_{\mathrm{T}}}{\sqrt{\mathrm{L}}}$.

By using the data from [23] and more detailed presentation in 25] we obtain $\bar{T} \approx 1.0394$ and $\mathrm{SE}_{\overline{\mathrm{T}}} \approx 0.0006$. This yields a $>60 \sigma$ violation, where $\sigma=\mathrm{SE}_{\overline{\mathrm{T}}}$. From the Chebyshev inequality

$$
P\left(\left|\bar{T}-\mu_{T}\right| \geq c\right) \leq \frac{\mathrm{SE}_{\overline{\mathrm{T}}}^{2}}{c^{2}}
$$

we estimate the confidence interval corresponding to the confidence level $99.95 \%$. We have $c \approx 0.027$.

$$
P\left(m_{T} \in[\bar{T}-0.027, \bar{T}+0.027]\right)=P\left(m_{T} \in[1.0124,1.0664]\right) \geq 0.9995 .
$$

Thus the confidence that can be placed in the result of the Vienna test is very high. The demonstrated violation of the (ratio-counts) $\mathrm{CH}$-inequality cannot be a matter of chance.

\section{The Vienna-test for the CH-inequality (in the ratio form): taking into account inten- sity drift}

In all previous considerations we assumed, as Eberhard originally [22], that the number of emitted pairs $N$ is constant during the experiment and does not depend on angles $\left(\alpha_{i}, \beta_{j}\right)$. In the real experiment, the intensity drift was very small [23]. In [25], a data analysis procedure was proposed being based on the following assumption:

$$
N\left(\alpha_{1}, \beta_{1}\right) / N\left(\alpha_{2}, \beta_{1}\right)=S_{o}^{B}\left(\alpha_{1}, \beta_{1}\right) / S_{o}^{B}\left(\alpha_{2}, \beta_{1}\right), \ldots
$$

Then one proceeds not simply with coincidence and single counts $n_{o o}, n_{o e}, \ldots$, $S_{o}^{A}, S_{o}^{B}$, but with their normalized values based on the above mentioned proportion of intensities. Denote normalized quantities as $\tilde{n}_{o o}, \tilde{n}_{o e}, \ldots, \tilde{S}_{o}^{A}, \tilde{S}_{o}^{B}$. For such normalized numbers of coincidences and singles, we can use the E-inequality:

$$
\tilde{n}_{o o}\left(\alpha_{1}, \beta_{1}\right)+\tilde{n}_{o o}\left(\alpha_{1}, \beta_{2}\right)+\tilde{n}_{o o}\left(\alpha_{2}, \beta_{1}\right)-\tilde{n}_{o o}\left(\alpha_{2}, \beta_{2}\right) \leq \tilde{S}_{o}^{A}\left(\alpha_{1}\right)+\tilde{S}_{o}^{B}\left(\beta_{1}\right) .
$$


And we jump directly to the CH-inequality for the total numbers of coincidences and singles (which is equivalent to the $\mathrm{CH}$-inequality for the rates):

$$
T^{\prime}=\frac{\tilde{n}_{o o}\left(\alpha_{1}, \beta_{1}\right)+\tilde{n}_{o o}\left(\alpha_{1}, \beta_{2}\right)+\tilde{n}_{o o}\left(\alpha_{2}, \beta_{1}\right)-\tilde{n}_{o o}\left(\alpha_{2}, \beta_{2}\right)}{\tilde{S}_{o}^{A}\left(\alpha_{1}\right)+\tilde{S}_{o}^{B}\left(\beta_{1}\right)} \leq 1 .
$$

Using data collected in [23] and [25], we get $\bar{T}^{\prime} \approx 1.0384$ with a violation $>60 \sigma$ which shows that also the experimental data taking into account intensity drift lead to the same amount of non-classical correlations.

\section{Concluding remark}

The statistical data collected in the Vienna-test [23, 25] violated statistically significantly not only the E-inequality, but equivalently also the $\mathrm{CH}-$ inequality for ratio of detection counts and, hence, the ratio-rate inequality, cf. [26], [27]. Thus, we can consider this experimental test as closing the detection loophole also with regard to the $\mathrm{CH}$-inequality in the ratio-form.

\section{Appendix: Statistical non-equivalence of (algebraically equivalent) linear and ratio test}

Let $X$ be a set of data sampled from realizations of some random variable $x$. Take two (for a moment arbitrary positive valued) functions, $J_{1}(x)$ and $J_{2}(x)$; set $J(x)=J_{1}(x)-J_{2}(x)$ and $T(x)=\frac{J_{2}(x)}{J_{1}(x)}$. Consider two tests for statistical data:

$$
\begin{aligned}
& J(x) \geq 0 ; \\
& T(x) \leq 1 .
\end{aligned}
$$

Suppose that the data $X$ showed $k \sigma_{J}$ violation of the first inequality, where $k$ is large. Thus violation of this inequality is significant. Our aim is to show that the same data can in principle show insignificant violation of the second inequality, say $\gamma \sigma_{T}$, where $\gamma$ is very small.

Suppose, for example, that the data $X$ was obtained as the result of measurements of a discrete random variable $x=x_{1}, x_{2}$, where $x_{1}, x_{2}$ are two arbitrary real numbers. It takes these values with probabilities $p_{1}$ and $p_{2}=1-p_{1}$. Here we need to set the values of all functions only in the two points, $x_{1}$ and $x_{2}$ :

$$
J_{1}\left(x_{i}\right)=A_{i}, J_{2}\left(x_{i}\right)=B_{i}, A_{i}, B_{i}>0, i=1,2 .
$$


We take $B_{i}=\left(1+\epsilon_{i}\right) A_{i}, \epsilon_{i}>0$. We have:

$$
\begin{gathered}
\mu_{J}=-\left(\epsilon_{1} A_{1} p_{1}+\epsilon_{2} A_{2} p_{2}\right), \sigma_{J}^{2}=p_{1} p_{2}\left(\epsilon_{1} A_{1}-\epsilon_{2} A_{2}\right)^{2} ; \\
\mu_{T}=1+\epsilon_{1} p_{1}+\epsilon_{2} p_{2}, \sigma_{J}^{2}=p_{1} p_{2}\left(\epsilon_{1}-\epsilon_{2}\right)^{2} ; \\
R_{J}=\frac{-\mu_{J}}{\sigma_{J}}=\frac{\epsilon_{1} A_{1} p_{1}+\epsilon_{2} A_{2} p_{2}}{\sqrt{p_{1} p_{2}}\left|\epsilon_{1} A_{1}-\epsilon_{2} A_{2}\right|} ; \quad R_{T}=\frac{\mu_{T}-1}{\sigma_{T}}=\frac{\epsilon_{1} p_{1}+\epsilon_{2} p_{2}}{\sqrt{p_{1} p_{2}}\left|\epsilon_{1}-\epsilon_{2}\right|} .
\end{gathered}
$$

By playing with parameters we want to make $R_{J}>>1$ and at the same time $R_{T}<<1$. First we set $\epsilon_{1}=\lambda \epsilon_{2}$, where $\lambda>1$. We have

$$
R_{T}=\sqrt{\frac{p_{1}}{p_{2}}}+\frac{1}{\sqrt{p_{1} p_{2}}(\lambda-1)} .
$$

To make the first term very small, we select $p_{1}=\delta^{2}<<1$, so $p_{2} \approx 1$; to make the second term very small, we select $\lambda$ in such a way that its denominator is very large, i.e., $\sqrt{p_{1} p_{2}}(\lambda-1)>>1$. Thus, for the model parameters satisfying conditions

$$
\delta<<1, \lambda>>1 / \delta
$$

the inequality (26) is violated insignificantly. We remark that, since the parameter $\epsilon_{2}$ has not yet been constrained, it is possible to make the absolute value of expectation very large, $\mu_{T}>>1$. We now want to make $R_{J}>>1$. We represent $A_{1}=a A_{2}$, and we have: $R_{J}==\frac{a \lambda p_{1}+p_{2}}{\sqrt{p_{1} p_{2}}|a \lambda-1|}$. Now we select $a$ as

$$
a \lambda-1>0
$$

Thus

$$
R_{J}=\frac{a \lambda p_{1}+p_{2}}{\sqrt{p_{1} p_{2}}(a \lambda-1)}=\sqrt{\frac{p_{1}}{p_{2}}}+\frac{1}{\sqrt{p_{1} p_{2}}(a \lambda-1)} .
$$

The first summand is negligibly small, see (27), it does not play any role in our considerations. The parameter $a$ has to be selected in such a way that the second summand will be very large. Thus $\sqrt{p_{1} p_{2}}(a \lambda-1)<<1$, or by taking into account (28) we obtain that $0<a \lambda-1<<1 / \sqrt{p_{1} p_{2}} \approx 1 / \delta$. Take very large natural number $k$. Suppose that

$$
a=1 / \lambda+1 / k \delta \lambda \text {. }
$$

Then $R_{J} \approx k 9$

\footnotetext{
${ }^{9}$ For example, set $\delta=0.1$, i.e., $p_{1}=0.01, p_{2}=0.99$. We remark that to get very small $R_{T}$ and at the same time very large $R_{J}$ the probability distribution of our model has to be strongly asymmetric. Then $\lambda=102$ guarantees that $R_{T}<0.2$. Finally, by setting $k=69$ we obtain that it is sufficient to take $a=0.0112$.
} 
Acknowledgment: We would like to thank all authors of the original experiment-paper [23] as they contributed to the data we used in this paper; especially, to M. Giustina whose numerous comments and suggestions essentially improved the paper. We would like to thank R. Gill, and R. Pettersson for discussions on statistical aspects of the paper and J.-A. Larsson and G. Adenier for discussions on the role of the fair sampling assumption in derivation of various versions of Bell inequality. This work was partially supported by MPNS COST Action MP1006, Fundamental Problems in Quantum Physics (I. Basieva), a visiting fellowship (A. Khrennikov) to Institute for Quantum Optics and Quantum Information, Austrian Academy of Sciences, and EU Marie-Curie Fellowship (S. Ramelow), PIOF-GA-2012-32985.

\section{References}

[1] J. S. Bell, Speakable and Unspeakable in Quantum Mechanics. Cambridge, Cambridge Univ. Press, 1987.

[2] P. M. Pearle, Phys. Rev. D 2 (8), 1418-1425 (1970).

[3] S. Pironio, A. Acin, S. Massar, A. Boyer de la Giroday, D. N. Matsukevich, P. Maunz, S. Olmschenk, D. Hayes, L. Luo, T. A. Manning, and C. Monroe, Nature 464, 1021-1024 (2010).

[4] H. Fürst, H. Weier, S. Nauerth, D. G. Marangon, Ch. Kurtsiefer, and H. Weinfurter, Optics Express 18, 13029-13037 (2010).

[5] A. Aspect, J. Dalibard, G. Roger, Phys. Rev. Lett. 49, 1804 (1982).

[6] A. Aspect, Three experimental tests of Bell inequalities by the measurement of polarization correlations between photons. Orsay (1983).

[7] G. Weihs, T. Jennewein, C. Simon, R. Weinfurter, and A. Zeilinger, Phys. Rev. Lett. 81, 5039-5043 (1998).

[8] M. A. Rowe, D. Kielpinski, V. Meyer, C. A. Sackett, W. M. Itano, C. Monroe and D. J. Wineland, Nature 409, 791-794 (2001).

[9] J. F Clauser, M.A. Horne, A. Shimony, R.A. Holt Phys. Rev. Lett. 23 (15), 880-884 (1969).

[10] J. F. Clauser and M. A. Horne, Phys. Rev. D 10, 526-535 (1974).

[11] J. F. Clauser and A. Shimony, Rep. Prog. Phys. 41, 1881-1927 (1978). 
[12] A. Fine, Synthese 50, 279-294 (1982).

[13] F. Selleri and A. Zeilinger, Found. Phys. 18, 1141 (1988).

[14] C. H. Thompson, Found. Phys. Lett. 9, 357 (1996).

[15] J.-A. Larsson, Phys. Rev. A, 57, 3304-3308 (1998).

[16] G. Adenier and A. Khrennikov, J. Phys. B: Atomic, Molecular Opt. Phys. 40 131-141 (2007).

[17] A. Khrennikov, Contextual approach to quantum formalism, Springer, Berlin-Heidelberg-New York, 2009.

[18] A. Khrennikov, J. Phys. A: Math. Theor. 45, 215301 (2012).

[19] A. E. Lita, A. J. Miller, and S. Woo Nam, Optics Express 16, 3032-3040 (2008).

[20] A. Shimony, Bell's Theorem, The Stanford Encyclopedia of Philosophy. E. N. Zalta (ed.), Stanford, 2012; URL = http://plato.stanford.edu/archives/win2012/entries/bell-theorem.

[21] N. D. Mermin, Techniques and and Ideas in Quantum Measurement Theory, edited by D.M. Greenberger (New York Academy of Science, New York, 1986), pp. 422428.

[22] Ph. H. Eberhard, Phys. Rev. A 477-750 (1993).

[23] M. Giustina, Al. Mech, S. Ramelow, B. Wittmann, J. Kofler, J. Beyer, A. Lita, B. Calkins, Th- Gerrits, S. Woo Nam, R. Ursin, and A. Zeilinger, Nature 497, 227-230 (2013).

[24] S. Ramelow, Conference Paper, Quantum Theory: Advances and Problems, A Marcus Wallenberg Symposium, Växjö, Sweden, June 10-13 (2013).

[25] J. Kofler, S. Ramelow, M. Giustina, and A.Zeilinger, On 'Bell violation using entangled photons without the fair-sampling assumption'. arXiv: 1307.6475 [quant-ph].

[26] P. Kwiat et al., Conference Paper, Quantum Information and Measurement, Rochester, New York (USA), Session Th3, June 17-20 (2013). 
[27] B. G. Christensen, K. T. McCusker, J. Altepeter, B. Calkins, T. Gerrits, A. Lita, A. Miller, L. K. Shalm, Y. Zhang, S. W. Nam, N. Brunner, C. C. W. Lim, N. Gisin, P. G. Kwiat, Phys. Rev. Letters, 111, 130406(2013).

[28] J. D. Gibbons and S. Chakraborti, Nonparametric Statistical Inference, 4th Ed. CRC, 2003.

[29] A. Aspect, Bell's Theorem: The Naive View of an Experimentalist. arXiv:quant-ph/0402001.

[30] J.-Å. Larsson, Quantum Paradoxes, Probability Theory, and Change of Ensemble. Linköping Univ. Press, Linköping (2000)

[31] G. Adenier, Local Realist Approach and Numerical Simulation of Non-

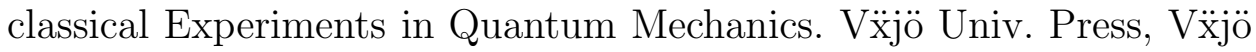
(2008).

[32] J.-A. Larsson, M. Giustina, J. Kofler, B. Wittmann, R. Ursin, S. Ramelow, Bell violation with entangled photons, free of the coincidence-time loophole, arxiv.org/abs/1309.0712

[33] A. Yu. Khrennikov, and I.V. Volovich, Local realism, contextualism and loopholes in Bell's experiments. In: Khrennikov, A.Yu. (ed) Foundations of Probability and Physics-2. Ser. Math. Model. 5, pp. 325-344. Växjö University Press, Växjö (2002).

[34] K. Hess and W. Philipp, Critique of Proofs, with and without Inequalities. In: Foundations of Probability and Physics-3, AIP Conf. Proc., vol. 750, ed. A. Khrennikov, Melville, AIP, 2005, pp. 516- 523.

[35] K. Hess and W. Philipp, EPL 57775 (2002).

[36] K. Hess, K. Michielsen and H. De Raedt, EPL 8760007 (2009).

[37] R. D. Gill, (2003), Time, finite statistics, and Bells fifth position, in: Foundations of Probability and Physics 2 (Vaxjo, 2002), vol. 5 of Math. Model. Phys. Eng. Cogn. Sci., pp. 179206, Växjö Univ. Press, Växjö, arXiv:quant-ph/0301059.

[38] R. D. Gill, G. Weihs, A. Zeilinger and M. Zukowski, EPL 61, 282 (2003).

[39] R. D. Gill, G. Weihs, A. Zeilinger, and M. Zukowski, Proc. Natl. Acad. Sci. USA 99, 1463214635 (2002). 
[40] R. D. Gill, Statistics, causality and Bell's theorem. Preprint, www.math.leidenuniv.nl/ gill/causality.pdf.

[41] T. Scheidl, R. Ursin, J. Kofler, S. Ramelow, X.-S. Ma, T. Herbst, L. Ratschbacher, A. Fedrizzi, N. K. Langford, T. Jennewein, and A. Zeilinger, Proc. Natl. Acad. Sci. USA 107, 19708-19713 (2010).

[42] M. Kupczynski, Phys. Lett. A 121, 205 (1987).

[43] A. Fine, Found. Phys. 19, N 5, 453-462 (1989).

[44] A. Khrennikov, Entropy 10, 19-32 (2008).

[45] G. Boole, Phil. Trans. Royal Soc. London 152, 225-242 (1862).

[46] G. Boole, An Investigation of the laws of thought. Dover Edition, New York (1958).

[47] N. N. Vorob'ev, Theor. Prob. Appl. 7 147-162 (1962).

[48] P. Tchebichef, Journal de mathématiques pures et appliquées 2(12), 177184 (1867).

[49] E. S. Fry and T. Walther, A Bell Inequality experiment based on molecular dissociation Extension of the Lo-Shimony proposal to $199 \mathrm{Hg}$ (nuclear spin 1/2) dimers, in Experimental Metaphysics, R.S. Cohen, M. Horne, and J. Stachel (eds.), Dordrecht-Boston-London: Kluwer, pp. 6171, 1997.

[50] J. G. Saw, M. C. K. Yang, and T. Ch. Mo, The American Statistician 38 (2), 130-132 (1984).

[51] A. Kabán, Statistics and Computing 22(2), 375385 (2012).

[52] H. Cramer, Mathematical Models of Statistics, 1946. 